\title{
Current role of Pipelle endometrial sampling in early diagnosis of endometrial cancer
}

\author{
Milan Milosav Terzic ${ }^{1,2,3}$, Gulzhanat Aimagambetova ${ }^{4}$, Sanja Terzic ${ }^{1}$, Melanie Norton ${ }^{5}$, Gauri Bapayeva ${ }^{2}$, \\ Simone Garzon ${ }^{6}$
}

${ }^{1}$ Department of Medicine, Nazarbayev University School of Medicine, Nur-Sultan, Kazakhstan; ${ }^{2}$ Clinical Academic Department of Women's Health, National Research Center of Mother and Child Health, University Medical Center, Nur-Sultan, Kazakhstan; ${ }^{3}$ Department of Obstetrics, Gynecology and Reproductive Sciences, University of Pittsburgh School of Medicine, Pittsburgh, PA, USA; ${ }^{4}$ Department of Biomedical Sciences, Nazarbayev University School of Medicine, Nur-Sultan, Kazakhstan; ${ }^{5}$ Department of Urogynaecology, Whittington Hospital, London, UK; ${ }^{6}$ Department of Obstetrics and Gynecology, University of Insubria, Varese, Italy

Contributions: (I) Conception and design: MM Terzic, G Bapayeva, S Garzon; (II) Administrative support: G Bapayeva, S Garzon; (III) Provision of study materials or patients: G Aimagambetova, S Terzic, M Norton; (IV) Collection and assembly of data: G Aimagambetova, S Terzic, M Norton; (V) Data analysis and interpretation: G Aimagambetova, S Terzic, M Norton; (VI) Manuscript writing: All authors; (VII) Final approval of manuscript: All authors.

Correspondence to: Milan Milosav Terzic. Department of Medicine, Nazarbayev University School of Medicine, office \#906, Zhanybek-Kerey khans Street, 5/1, Nur-Sultan 010000, Kazakhstan. Email: milan.terzic@nu.edu.kz.

\begin{abstract}
Endometrial cancer is the most common gynecologic cancer in developed countries. The most prevalent, however not pathognomonic symptom of the disease is abnormal uterine bleeding. The diagnosis of endometrial cancer is based on the histologic results of endometrial sampling. Endometrial biopsy could be obtained using different modalities: hysteroscopy-directed endometrial biopsy, uterine curettage or office endometrial biopsy. Outpatient endometrial biopsy using different devices for the evaluation of abnormal uterine bleeding is gaining popularity. The most popular office-based device for endometrial sampling procedure is the Pipelle device. Currently, Pipelle endometrial sampling is widely used to diagnose endometrial cancer in women with abnormal uterine bleeding and/or postmenopausal bleeding. The method became very useful due to easiness and simplicity of the procedure, availability of a device, as well as high sensitivity in detecting endometrial cancer. Many studies compared the validity and accuracy of Pipelle biopsy with dilation and curettage in the detection of various endometrial pathologies. Published results state that Pipelle biopsy and uterine curettage are almost equally reliable in the evaluation of endometrial pathologies. Moreover, Pipelle biopsy appears more beneficial as it does not require hospital admission and anaesthesia. However, it is proven the Pipelle technique has a limited capacity to identify endometrial polyps, and some authors, based on their study, claim that dilation and curettage is a more reliable method in terms of correlation with the final histological results. In addition, there are many factors affecting the efficiency of the endometrial biopsy. Failure to get samples that are adequate for histological examination is one of the problems associated with Pipelle sampling. The above mentioned contradictory conclusions by different researchers and lack of guidance to avoid inadequate sampling present the demand for further studies on the comparison of Pipelle biopsy and uterine curettage efficiency and accuracy.
\end{abstract}

Keywords: Dilation and curettage; early diagnosis; endometrial cancer; endometrial sampling; Pipelle biopsy

Submitted Feb 20, 2020. Accepted for publication Apr 07, 2020.

doi: $10.21037 /$ tcr.2020.04.20

View this article at: http://dx.doi.org/10.21037/tcr.2020.04.20 


\section{Introduction}

Adenocarcinoma is the most common form of endometrial cancer (EC) and it is the fifth most common malignant disorder among women worldwide and the fourth most common female cancer in the US (1-6). In 2012 EC was diagnosed in 527,600 women in the globe with the mortality rate 1.7 to 2.4 per 100,000 women (7). EC develops in about 142,000 women worldwide each year, with the mortality rate of 42,000 patients (1). In the United States EC incidence was 61,380 new uterine cancer cases in 2017, with almost 11,000 deaths from the disease $(3,4)$. Throughout the last 20 years, the incidence and mortality rate for EC has increased by more than $100 \%(2,8)$. Moreover, the incidence varies $\sim 10$-fold worldwide with estimated agestandardized rates of 15 per 100,000 women and higher in 2018 in Europe and North America (developed countries). However, lower incidences were reported in parts of Africa and Asia, for example, in Algeria (ASR $=2.2 / 100,000$ ) or India (ASR $=1.9 / 100,000)(8-10)$.

Unfortunately, the incidence is rising with an increase in life expectancy. This increased number has been associated with obesity and physical inactivity (1). EC was diagnosed in a mean age of 63 years, and $90 \%$ of patients are older than 50 years. Diagnosis of EC was established before menopause only in $20 \%$ of patients $(2,11)$.

There have been several identified risk factors found to be associated with the increasing risk of EC development: postmenopausal age, long-term exposure to unopposed estrogens, metabolic syndrome (obesity, diabetes), years of menstruation, nulliparity, history of breast cancer, long-term use of tamoxifen, family history $(1,2,7,10,12)$. However, some factors have been found to reduce the risk: grand multiparity, smoking, oral contraceptive use, physical activity, and diet of some phytoestrogens $(1,2,10)$.

Post-menopausal bleeding is the most common presenting complaint of patients with EC $(1,2,13)$. However, this bleeding facilitates making an appropriate diagnosis (1). If postmenopausal patient experiences bleeding, the probability of EC is $5-10 \%$, while chances increase with age and risk factors (1). EC is diagnosed at an early stage in almost $75 \%$ of postmenopausal women, which presents a foundation for successful treatment (2). All postmenopausal women with vaginal bleeding, especially associated with the above mentioned risk factors for EC, should undergo further diagnostic endometrial assessment $(1,2)$. Evaluation is supposed to be done first by ultrasound scan and then by endometrial biopsy (2). Endometrial tissue sampling has been the keystone examination for the diagnosis of EC and contributes to the patients' management (14). Published literature shows that, in spite of the higher rate of accompanying diseases, advanced age patients can also benefit from standard treatment to manage their gynaecological cancers $(11,15)$.

\section{Overview on diagnostic modalities for detection of endometrial carcinoma}

EC is the most commonly diagnosed histologically from endometrial tissue. Endometrial biopsy, as the primary and basic diagnostic method for different endometrial pathologies, could be obtained utilizing different modalities $(1,16)$. In order to establish the right diagnosis, there is a need for a sufficient amount and adequate quality of the endometrial specimen. There are numbers of modalities that serve to obtain endometrial samples including classic dilation and curettage procedure (D\&C), hysteroscopy, Pipelle sampling, biopsy with Vabra Z-sampler, MiMark cell sampler, Isaacs cell sampler, Gynoscann device, Endorette, Tao Brush, SAP-1 device, etc. (16-19). Nowadays, sampling with miniature endometrial biopsy outpatient device, such as Pipelle, is the first-choice approach $(1,16)$. However, none of the above-mentioned methods allow scraping the whole uterine cavity; therefore, it is upon the clinician to choose the appropriate technique for each particular patient (16).

The invention of the D\&C is generally attributed to Recamier in 1844 (20,21). Over 170 years have passed since then and this procedure has become popular and for many years, it has been the 'gold standard' method for endometrial sampling $(21,22)$. It has been estimated that the uterine cavity abrasion allows for the examination of $50-60 \%$ of the lining surface, while the percentage of nondiagnostic results reaches $3 \%(16,23)$. However, uterine cavity scrapings were done with the additional risks of general anaesthesia and complications, such as infections, bleeding and uterine perforation in overall $60 \%$ of cases (23). $\mathrm{D} \& \mathrm{C}$ does not guarantee obtaining sufficient specimen from the uterine cavity, despite increasing the invasiveness of scrapings. D\&C was found to be an expensive procedure, especially in the USA, with a high rate of complications and significant social impact $(20,22,24)$. These issues with the $\mathrm{D} \& \mathrm{C}$ procedure pushed physicians to find and implement a safer, less invasive, more accurate and easily applicable method for endometrial sampling. Following the demand, a lot of new technical approaches for endometrial sampling 
were introduced over the past decades. Techniques using jet-irrigation and cell-samplers were found to be of use for diagnostic purposes, although relying on proper cytological analysis/interpretation (20).

Another possible approach to get an endometrial sample is to use the vacuum principle. Currently, available vacuum curettage devices enable endometrial sampling in outpatient settings without general anaesthesia.

Tissue aspiration by using an air-suction pump was first described in 1849 by James (25). Vacuum curettage by Vabra aspiration of the uterus for the endometrial disease was introduced by Jensen and Jensen in 1968 (26). Vabra is an acronym for Vacuum ABRasio and Aspiration (25). The Vabra aspiration system is a stainless steel cannula of $4.2 \mathrm{~mm}$ in diameter attached to a portable electric vacuum pump. Since its invention, there have been around 30 studies confirming excellent patient acceptance and diagnostic value with the Vabra aspirator (22). However, later it was found that utilization of the Vabra method is not very good at detecting polyps and could miss early cancers (20). Nevertheless, it was found to be an acceptable alternative to $\mathrm{D} \& \mathrm{C}$ procedure.

Utilization of the Gynoscann method comprises a disposable, plastic curette of $3 \mathrm{~mm}$ in diameter, consisting of two flexible wings attached to the end of a flexible rod and contained in a thin tube (27). However, according to comparative studies, Gynoscann cannot replace D\&C without the risk of overlooking significant pathology $(17,27)$. The other miniature endometrial biopsy device "Uterine Explora Curetter" was proven to be superior to Gynoscann in diagnosing neoplasia of the endometrium. Gynoscann was found to have lower diagnostic accuracy than conventional D\&C (17).

The other relatively new endometrial sampling device Endorette (Medscand AB, Sweden) is similar to Pipelle in its slightly smaller diameter construction $(3.1 \mathrm{~mm})$ but has a larger number of holes/openings around the circumference of the tip, in order to enhance its capacity for collecting adequate endometrial samples (18).

Since the beginning of the ' 80 s, hysteroscopies have been a way to provide clinicians with direct visualization of the cervical canal and the uterine cavity (28). Hysteroscopy is acknowledged to be very useful and informative procedure of uterine cavity assessment for endometrial and intrauterine abnormalities associated with both preand postmenopausal abnormal uterine bleeding (29,30). Nowadays, the investigation is usually done in an outpatient setting. Currently, this technique is associated with less discomfort compared to the traditional approach comprising the use of a speculum and tenaculum (29). In the USA 15$25 \%$ of gynecologists perform an office hysteroscopy (30). Endometrial sampling should be done in all postmenopausal bleeding women with an endometrial thickness of $\geq 4 \mathrm{~mm}$ $(1,30)$. However, in $11 \%$ of cases, EC is missed due to blind sampling alone. Additional 7\% of EC cases are with failed endometrial sampling (either due to inadequate tissue obtained or inability to approach the uterine cavity) (29). Tissue biopsy facilitated by hysteroscopy improves the accuracy of the diagnosis of EC $(28,30)$.

The Tao Brush was invented in 1993, and approved by the Food and Drug Administration (19,31). The brush should be inserted through the cervical canal to the level of the uterine fundus. After that, the brush should be rotated $360^{\circ} 3-5$ times to collect an appropriate amount of endometrial cells. The Tao Brush in found to be very good for an outpatient clinical setting, without the need for anaesthesia (19). Specimen obtaining satisfaction with the Tao Brush was $89.9 \%$ to $100 \%$, while the pathological accuracy was $91 \%$ to $96 \%(19,32)$. Some researchers suggest using Tao Brush sampling as an alternative or additional sampling device in postmenopausal women, as it offers advantages over the use of Pipelle (33).

The most recent from the above-mentioned techniques, SAP-1 device, was patented and approved to be used in China in 2001 (19). The sheath of this device is $25 \mathrm{~cm}$ in length and $3 \mathrm{~mm}$ in diameter. SAP-1 is becoming a reliable method for screening EC and its precursors, especially as it shows high reliability: sensitivity $73 \%$, specificity $95.8 \%$, positive predictive value (PPV) $75 \%$ and negative predictive value (NPV) $95.3 \%(19,34)$.

Over the years, outpatient endometrial biopsy for abnormal uterine bleeding is gaining popularity (16). Nevertheless, some clinicians remain reluctant to perform ambulatory endometrial sampling. The main reason behind this opposition is due to patient discomfort. However, new techniques have significantly reduced this complaint $(1,16)$.

\section{Pipelle endometrial sampling}

Endometrial pathology has led to the development of multiple instruments designed for endometrial biopsy. However, each still presents some disadvantages of routine cancer screening. The most popular vacuum aspiration device for office sampling procedure is the Pipelle device invented in 1984 by Cornier E., Paris, France $(1,35,36)$. Currently, Pipelle endometrial biopsy is widely used in 
women with abnormal uterine bleeding or postmenopausal bleeding (37). The method became very useful due to its simplicity as well as high sensitivity in detecting EC $(16,36)$.

\section{Pipelle sampling device and its modifications}

The Pipelle de Cornier is a flexible polypropylene tube, with an external diameter of $3.1 \mathrm{~mm}$ and an internal diameter of $2.6 \mathrm{~mm}$. The sheath measures $23.5 \mathrm{~cm}$ in length and has a soft endouterine end. There is a perforation, $2.4 \mathrm{~mm}$ in diameter, near the endouterine end of the sheath (35). During the removal of an internal piston, negative pressure is created and endometrial tissue comes into the cannula. The uterine mucosa is then clearly visible within the sheath. Bleeding is minimal or, most often, entirely absent. In the cited study, two hundred sixty biopsies have been performed, with no uterine infection or perforation (35). The Pipelle device, which does not require a suction pump, is less expensive, more convenient, and is associated with less patient discomfort than the other endometrial sampling methods $(16,38)$.

The other modifications were created based on the genuine device like Pipelle $\mathrm{H}$ device, Pipelle Mark II.

Pipelle $\mathrm{H}$ device was developed at the Royal Free Hospital in London for histological sampling during routine hysteroscopies. The Pipelle H optimizes endometrium sampling during the procedure of diagnostic hysteroscopy $(37,38)$. The Pipelle $\mathrm{H}$ is twice longer $(50 \mathrm{~cm})$, but has the same external and internal diameters as the genuine Pipelle de Cornier (29). The device enables appropriate suction to collect a sufficient volume of endometrial tissue. Handling with Pipelle $\mathrm{H}$ is faster and with less patient discomfort than the use of a standard Pipelle (29,39).

Pipelle Mark II (Laboratoire CCD, Paris, France) is the only device providing samples for both histology and cytology in one single attempt/procedure; and it is a possible explanation of its high efficacy. The sampling technique is identical to the original Pipelle one (37). Pipelle Mark II decreases the rate of false-negative results for EC and is particularly useful in postmenopausal women and in women with endometrial atrophy (37).

\section{Current role of Pipelle biopsy in practice}

\section{Reliability and validity of Pipelle biopsy}

Many researchers have already investigated the performance rate of a Pipelle biopsy. In one of the early studies, the technique was described as adequate for analysis in $97 \%$ of patients (40). In this study, malignancy was detected by biopsy in 54 of 65 patients, with a sensitivity of $83 \% \pm 5 \%$ (mean \pm SD). The Pipelle endometrial sampling was found to be an effective device for evaluating patients at risk of endometrial cancer (40). However, small tumours of the endometrium may be left undetected. In comparison with D\&C, Pipelle device has become superior enabling both outpatients sampling and decreasing the cost of the service (16).

Many studies compared the validity and accuracy of Pipelle biopsy with $\mathrm{D} \& \mathrm{C}$ in the detection of various endometrial abnormalities.

One huge meta-analysis evaluated the value of Pipelle biopsy for the diagnosis of atypical hyperplasia and EC (23). This analysis presents its sensitivity and specificity of $81-99 \%$ and $98 \%$, respectively. The accuracy is better in symptomatic bleeding and postmenopausal women and for the diagnosis of EC compared to atypical endometrial hyperplasia $(1,23,41)$. The detection rate for EC was higher in postmenopausal women compared with premenopausal women. The Pipelle was the best device in both postmenopausal and premenopausal women, with detection rates of $99.6 \%$ and $91 \%$, respectively (23). For the detection of atypical hyperplasia, there was only one study that reported results on postmenopausal women, and this study did not provide the possibility of subgroup analysis. According to the cited study, the Pipelle has a sensitivity of $81 \%$ and specificity of more than $98 \%$ (23). Overall, in this research, endometrial biopsy with the Pipelle was reported to be better than other endometrial sampling techniques in the detection of both EC and atypical hyperplasia. The accuracy of the Pipelle was shown to be higher in postmenopausal women compared with premenopausal women (23).

Several studies have shed some light on the validity and adequacy of Pipelle endometrial sampling in comparison with D\&C and hysterectomy specimens (21). With respect to Pipelle biopsies' reliability in the determination of endometrial polyps, the method was confirmed to have sensitivity $12.5 \%$, specificity $100 \%$, positive predictive value (PPV) $100 \%$ and negative predictive value (NPV) $88.7 \%$ (21). If compared, $\mathrm{D} \& \mathrm{C}$ procedure in identifying endometrial polyps shows higher sensitivity $(87.5 \%)$ and NPV (98\%), but lower specificity (94.8\%) and PPV (70\%). In the same study, with respect to hyperplasia without atypia, the sensitivity was $23.5 \%$, the SP was $100 \%$, the PPV was $100 \%$ and the NPV was $78 \%$. D\&C in terms of hyperplasia without atypia reveals the sensitivity $55.6 \%$, the specificity $95.8 \%$, the PPV $83.3 \%$, and the NPV $85.2 \%$. When researchers compared Pipelle biopsy and 
Table 1 Comparison of Pipelle biopsy and D\&C reliability in detection of endometrial pathology

\begin{tabular}{|c|c|c|c|c|c|c|c|c|}
\hline Pathology & \multicolumn{4}{|c|}{ Pipelle biopsy } & \multicolumn{4}{|c|}{ D\&C procedure } \\
\hline Endometrial polyp & 12.5 & 100 & 100 & $88.7-96.1$ & 87.5 & 94.8 & $70 \%$ & $98 \%$ \\
\hline Hyperplasia without atypia & $23-67$ & $88.8-100$ & $42.9-100$ & 78-95.5 & $55.6-92.3$ & $95.8-100$ & $83.3-100$ & $85.2-98.1$ \\
\hline Hyperplasia with atypia & $50-100$ & $98-100$ & $33.3-100$ & $95-100$ & $83-84$ & 98.3 & 75 & 98.3 \\
\hline
\end{tabular}

SN, sensitivity; SP, specificity; PPV, positive predictive value; NPV, negative predictive value.

hysterectomy histological results with respect to hyperplasia with atypia, the sensitivity of the Pipelle was $50 \%$, the specificity $100 \%$, the PPV and the NPV were $100 \%$ and $95 \%$, respectively (21). In the same study, the sensitivity of $\mathrm{D} \& \mathrm{C}$ procedure in the identification of hyperplasia with atypia was $83.3 \%$, the specificity was $98.3 \%$ and the NPV was $98.3 \%$.

According to other recent investigations, the concordance rate of histologic results between Pipelle biopsy and hysterectomy was $62 \%$ and between D\&C and hysterectomy was $67 \%$ (42). In the cited study, the sensitivity of Pipelle biopsy and D\&C for detecting simple hyperplasia was $41.7 \%$ and $45 \%$, respectively, and for detecting atypia was $71.4 \%$ for both techniques (42). The $\mathrm{SN}$ of detecting atrophic endometrial tissue was significantly higher in the $\mathrm{D} \& \mathrm{C}$ group at $80 \%$ compared to $37.5 \%$ in the Pipelle biopsy group $(\mathrm{P}=0.030)$. All other parameters were similar in both groups. Based on their results authors concluded that Pipelle biopsy and D\&C were evenly effective as a diagnostic approach of endometrial pathologies (42). However, none of the methods were adequate for detecting focal endometrial pathologies and endometrial hyperplasia. From the other side, both Pipelle and D\&C provided a sample for a reliable diagnosis of atypia.

Reported sensitivity and specificity were similarly demonstrated in diagnosing atypia and EC (43). The cited study aimed to identify the rate of endometrial sampling failure in Pipelle endometrial biopsy in comparison to the $\mathrm{D} \& \mathrm{C}$ as well as duration and costs. Pipelle diagnostic accuracy has been reported over $97 \%$, so the failure rate in this study was below 5\%, while the sensitivity of Pipelle for the detection of endometrial atrophy was reported below $50 \%$. The duration of procedure and cost were lower in Pipelle sampling than in D\&C. The authors concluded that due to the high adequacy of histopathology specimen (except atrophic endometrium), low failure rate, low duration of sampling and cost, Pipelle biopsy was recommended as an appropriate alternative for diagnostic uterine curettage (43).

Other researchers using Pipelle biopsy with a hysteroscopic examination of women with endometrial polyps found the PPV of the method was $56.3 \%$ (44). The reliability was higher in the postmenopausal women (72.7\%) compared to premenopausal women (53.7\%). Hysteroscopy remains to be a very reliable diagnostic tool for endometrial polyps, especially in infertility patients (45).

The most recent study investigated the reliability of the Pipelle technique by comparing the histopathology report obtained by Pipelle biopsy with the hysterectomy specimen (46). In this study, the overall concordance rate was $63.8 \%$. The sensitivity, specificity, PPV and NPV, and accuracy of Pipelle biopsy for endometrial hyperplasia was $64.2 \%, 88.8 \%, 94.1 \%, 85.5 \%$, and $47.3 \%$ and for endometrial carcinoma was $75 \%, 100 \%, 100 \%, 97.9 \%$, and $98 \%$, respectively $(\mathrm{P}=0.001)$ (46). Available up-to-date data $(21,23,42,43,46)$ on the reliability of the Pipelle biopsy technique are summarized in Table 1.

As it is clearly seen from Table 1, Pipelle and D\&C are almost equally reliable in the evaluation of endometrial pathologies. However, the $\mathrm{D} \& \mathrm{C}$ procedure has many disadvantages like a uterine perforation in $0.6-1.3 \%$, infection in $0.3-0.5 \%$, unexpected hemorrhage in $0.4 \%$ of the cases (45). There are also complications associated with general anaesthesia or hospital admission $(1,2,16,46)$. In comparison, Pipelle biopsy appears more beneficial as it does not require hospital admission and anaesthesia. However, Pipelle biopsy has a limited capacity to identify endometrial polyps (Table 1). Therefore, in cases with suspected focal endometrial lining abnormalities, methods allowing biopsy with visualization (such as hysteroscopy) were suggested to be used (46).

However, some authors, based on their study, still state that $\mathrm{D} \& \mathrm{C}$ is more reliable in terms of correlation 
with the final histological results to Pipelle biopsies $(14,47)$. Moreover, D\&C could help to remove the tumour completely if it is a focal malignancy (14). The above mentioned contradictory conclusions by different researchers present demand for further studies to evaluate D\&C and Pipelle biopsy accuracy.

\section{Factors affecting the Pipelle biopsy efficiency and failure rate}

Currently, there is still lack of data about factors influencing the effectiveness of Pipelle biopsy and D\&C. Failure to obtain adequate samples for histological examination is one of the major issues associated with the Pipelle procedure $(46,48)$. Identification of factors that may have an impact on the efficacy of endometrial sampling could help in the selection of the diagnostic technique for each particular patient (16).

In the early German study, researchers compared outpatient endometrial biopsy utilizing the Pipelle sampler with conventional $\mathrm{D} \& \mathrm{C}$ in patients with abnormal uterine bleeding (49). Endometrial samples were obtained with the Pipelle curette in 172 patients, and with D\&C in 97 patients. By the end of the study, $98.8 \%$ of the Pipelle biopsy attempts were successfully completed with sufficient material for histological assessment obtained in $90.6 \%$. In the group of $\mathrm{D} \& \mathrm{C}$ biopsy only $68 \%$ of procedures were successful $(\mathrm{P}<0.001)$. In postmenopausal patients, the researchers succeeded to obtain adequate specimens by the Pipelle technique in $84.1 \%$, while by D\&C in only $45.8 \%$ of patients (49). The investigation of 45 cases, a comparison between histological diagnosis of endometrial tissue obtained by Pipelle sampling with that available following $\mathrm{D} \& \mathrm{C}$ or even hysterectomy was done. The diagnosis from both of these methods was identical in $95.5 \%$. Interestingly, the other 2 cases led to misdiagnoses of atrophic endometrium (49).

According to different studies, the amount of inadequate Pipelle specimens varies between $7 \%$ and $40 \%(16,21,47,48,50)$. Difficulties in getting samples are particularly true in postmenopausal women. Around $6 \%$ of postmenopausal women with failed diagnostic biopsy after repeated endometrial samplings are found to have clinically important endometrial changes $(41,50)$. In this view, different factors have an impact on the procedure success: (I) patient related-factors like prior cervical procedures, genital anomalies, obesity; and (II) provider related factors like the inability to access endometrium, inadequate sample, physicians' experience, etc. $(16,43)$. According to previous study reports, failure to get an adequate sample could be influenced by the endometrial thickness (48). According to the cited author, if the endometrial thickness is $<5 \mathrm{~mm}$, only $27 \%$ of the specimens will be adequate for histological analysis.

A more recent study of factors affecting Pipelle biopsy and its failure rate, revealed that Pipelle failed in $22.89 \%$, where in $17.39 \%$ it happened due to inability to reach the endometrium, in $80.43 \%$ were inadequate samples, and in $2.18 \%$ was due to unknown reasons (51). Provider and patient-related factors were found to be related with sampling failure: postmenopausal bleeding as biopsy indication (OR 7.41, 95\% CI: 2.27-24.14); a history of prior biopsy failure (OR 23.87, 95\% CI: 3.76-151.61); and "provider type" factor (physician vs. mid-level provider) (OR 9.152, 95\% CI: 2.49-33.69) (50).

Another study aimed to investigate the factors affecting the quality of specimens obtained for histological analysis among women who underwent Pipelle endometrial biopsy and $\mathrm{D} \& \mathrm{C}$, including investigators' professional experience (16). According to the results, the main factors that influenced the accuracy of Pipelle sampling were patient's age and menopausal status. Inadequate samples were found in $7.8 \%$ of premenopausal and $40.2 \%$ of postmenopausal women.

The other similar study aimed to check the efficacy of Pipelle biopsy by the adequacy of the sample obtained (46). The number of inadequate endometrial samples obtained in the research accounted for $22.1 \%$, with an adequacy rate of $77.9 \%$, which was comparable to a study conducted earlier $(21,46,48)$. The authors concluded that the Pipelle technique is an effective device in obtaining adequate samples.

The diagnostic value of Pipelle biopsy for EC depends on many factors. Its diagnostic accuracy could decrease significantly when a few influencing factors overlap (15). In the currently published literature available, there is no suggestion on how to improve Pipelle failure rate.

\section{Conclusions}

Endometrial abnormalities are frequent problems in gynecologic practice, especially in premenopausal and postmenopausal women. Many endometrial sampling techniques are employed to diagnose endometrial pathologies for patients with or without abnormal uterine bleeding. Pipelle endometrial sampling is a simple, inexpensive procedure that gives minimal discomfort and pain to the patient, thus improving the patient's satisfaction and compliance. The diagnostic effectiveness and reliability of the Pipelle device in endometrial sampling depend on many patients' and providers' related factors 
like age, menopausal status, BMI, the indications for the procedure, and physician's experience. Some authors still state that $\mathrm{D} \& \mathrm{C}$ is more reliable than a Pipelle biopsy in terms of correlation with the final histological results. The above mentioned contradictory conclusions by different researchers raised the need for further studies on the comparison of $\mathrm{D} \& \mathrm{C}$ and Pipelle biopsy reliability. Additionally, the development of Pipelle biopsy guidance will reduce sampling failures and improve the value of the technique, already confirmed as useful for endometrial cancer diagnosis in daily clinical practice.

\section{Acknowledgments}

Funding: This study was supported by the Nazarbayev University Grant Number 110119FD4540, 2019-2022.

\section{Footnote}

Provenance and Peer Review: This article was commissioned by the Guest Editor (Antonio Simone Laganà) for the series "Endometrial Cancer" published in Translational Cancer Research. The article was sent for external peer review organized by the Guest Editor and the editorial office.

Conflicts of Interest: All authors have completed the ICMJE uniform disclosure form (available at http://dx.doi. org/10.21037/tcr.2020.04.20). The series "Endometrial Cancer" was commissioned by the editorial office without any funding or sponsorship. The authors have no conflicts of interest to declare.

Ethical Statement: The authors are accountable for all aspects of the work in ensuring that questions related to the accuracy or integrity of any part of the work are appropriately investigated and resolved.

Open Access Statement: This is an Open Access article distributed in accordance with the Creative Commons Attribution-NonCommercial-NoDerivs 4.0 International License (CC BY-NC-ND 4.0), which permits the noncommercial replication and distribution of the article with the strict proviso that no changes or edits are made and the original work is properly cited (including links to both the formal publication through the relevant DOI and the license). See: https://creativecommons.org/licenses/by-nc-nd/4.0/.

\section{References}

1. Amant F, Moerman P, Neven P, et al. Endometrial cancer. Lancet 2005;366:491-505.

2. Braun MM, Overbeek-Wager EA, Grumbo RJ. Diagnosis and Management of Endometrial Cancer. Am Fam Physician 2016;93:468-74.

3. Koh WJ, Abu-Rustum NR, Bean S, et al. Uterine Neoplasms, Version 1.2018, NCCN Clinical Practice Guidelines in Oncology. J Natl Compr Canc Netw 2018;16:170-99.

4. Casarin J, Bogani G, Serati M, et al. Presence of Glandular Cells at the Preoperative Cervical Cytology and Local Recurrence in Endometrial Cancer. Int J Gynecol Pathol 2020;39:522-8.

5. Franchi M, Garzon S, Zorzato PC, et al. PET-CT scan in the preoperative workup of early stage intermediate- and high-risk endometrial cancer. Minim Invasive Ther Allied Technol 2020;29:232-9.

6. Siegel RL, Miller KD, Jemal A. Cancer statistics, 2019. CA Cancer J Clin 2019;69:7-34.

7. Moore K, Brewer MA. Endometrial Cancer: Is This a New Disease? Am Soc Clin Oncol Educ Book 2017;37:435-42.

8. Lortet-Tieulent J, Ferlay J, Bray F, et al. International Patterns and Trends in Endometrial Cancer Incidence, 1978-2013. J Natl Cancer Inst 2018;110:354-61.

9. Cignini P, Vitale SG, Laganà AS, et al. Preoperative workup for definition of lymph node risk involvement in early stage endometrial cancer: 5-year follow-up. Updates Surg 2017;69:75-82.

10. Fortner RT, Hüsing A, Dossus L, et al. Theoretical potential for endometrial cancer prevention through primary risk factor modification: Estimates from the EPIC cohort. Int J Cancer 2020;147:1325-33.

11. Vitale SG, Capriglione S, Zito G, et al. Management of endometrial, ovarian and cervical cancer in the elderly: current approach to a challenging condition. Arch Gynecol Obstet 2019;299:299-315.

12. Terzic M, Norton M, Terzic S, et al. Fertility preservation in endometrial cancer patients: options, challenges and perspectives. Ecancermedicalscience 2020;14:1030.

13. Andrijasevic S, Dotlic J, Arsenovic N, et al. Differences in endometrial carcinoma presentations and characteristics in pre- and postmenopausal women. Srp Arh Celok Lek 2019;147:692-98.

14. Vitale SG, Rossetti D, Tropea A, et al. Fertility sparing surgery for stage IA type I and G2 endometrial cancer in 
reproductive-aged patients: evidence-based approach and future perspectives. Updates Surg 2017;69:29-34.

15. Laganà AS, La Rosa VL, Rapisarda AM, et al. Comment on: "Needs and priorities of women with endometrial and cervical cancer". J Psychosom Obstet Gynaecol 2017;38:85-6.

16. Piatek S, Warzecha D, Kisielewski F, et al. Pipelle biopsy and dilatation and curettage in clinical practice: are factors affecting their effectiveness the same? J Obstet Gynaecol Res 2019;45:645-51.

17. Kufahl J, Pedersen I, Sindberg Eriksen P, et al. Transvaginal ultrasound, endometrial cytology sampled by Gynoscann and histology obtained by Uterine Explora Curette compared to the histology of the uterine specimen. A prospective study in pre- and postmenopausal women undergoing elective hysterectomy. Acta Obstet Gynecol Scand 1997;76:790-6.

18. Moberger B, Nilsson S, Palmstierna S, et al. Amulticenter study comparing two endometrial sampling devices Medscand Endorette TM and Pipelle De Cornier R. Acta Obstet Gynecol Scand 1998;77:764-9.

19. Du J, Li Y, Lv S, et al. Endometrial sampling devices for early diagnosis of endometrial lesions. J Cancer Res Clin Oncol 2016;142:2515-22.

20. Seamark CJ. The demise of the D\&C. J R Soc Med 1998;91:76-9.

21. Kazandi M, Okmen F, Ergenoglu AM, et al. Comparison of the success of histopathological diagnosis with dilatation-curettage and Pipelle endometrial sampling. J Obstet Gynaecol 2012;32:790-4.

22. Naim NM, Mahdy ZA, Ahmad S, et al. The Vabra aspirator versus the Pipelle device for outpatient endometrial sampling. Aust N Z J Obstet Gynaecol 2007;47:132-6.

23. Dijkhuizen FP, Mol BW, Brölmann HA, et al. The accuracy of endometrial sampling in the diagnosis of patients with endometrial carcinoma and hyperplasia: a meta analysis. Cancer 2000;89:1765-72.

24. Xie B, Qian C, Yang B, et al. Risk Factors for Unsuccessful Office-Based Endometrial Biopsy: A Comparative Study of Office-Based Endometrial Biopsy (Pipelle) and Diagnostic Dilation and Curettage. J Minim Invasive Gynecol 2018;25:724-9.

25. Helmerhorst TJM, Franke HR, Risse EKJ, et al. Endocervical curettage by Vabra aspiration as part of colposcopic evaluation. Gynecol Oncol 1990;36:312-6.

26. Jensen JA, Jensen JG. Abrasion of the uterine mucosa by aspiration. Preliminary report. Ugeskr Laeger
1968;130:2124-7.

27. Schei B, Bang TF, Halgunset J, et al. Microcurettage sampling of the endometrium for histopathological examination - simpler but not safe? Comparison of endometrial histopathology in samples obtained by a disposable mechanical curette and by traditional curettage. Acta Obstet Gynecol Scand 1994;73:497-501.

28. Campo R, Santangelo F, Gordts S, et al. Outpatient hysteroscopy. Facts Views Vis Obgyn 2018;10:115-22.

29. Dacco' MD, Moustafa M, Papoutsis D, et al. An audit of using the H Pipelle for endometrial sampling at outpatient hysteroscopy and literature review comparison with the Pipelle de Cornier. Eur J Obstet Gynecol Reprod Biol 2012;165:299-301.

30. Salazar CA, Isaacson KB. Office Operative Hysteroscopy: An Update. J Minim Invasive Gynecol 2018;25:199-208.

31. Tao LC. Direct intrauterine sampling: the IUMC Endometrial Sampler. Diagn Cytopathol 1997;17:153-9.

32. Maksem J, Sager F, Bender R. Endometrial collection and interpretation using the Tao brush and the CytoRich fixative system: a feasibility study. Diagn Cytopathol 1997;17:339-46.

33. Williams AR, Brechin S, Porter AJ, et al. Factors affecting adequacy of Pipelle and Tao Brush endometrial sampling. BJOG 2008;115:1028-36.

34. Wen J, Chen R, Zhao J, et al. Combining endometrium sampling device and SurePath preparation to screen for endometrial carcinoma: a validation study. Chin Med J (Engl) 2015;128:648-53.

35. Cornier E. The Pipelle: A disposable device for endometrial biopsy. Am J Obstet Gynecol 1984;148:109-10.

36. Piątek S, Panek G, Wielgoś M. Assessment of the usefulness of pipelle biopsy in gynecological diagnostics. Ginekol Pol 2016;87:559-64.

37. Polena V, Mergui JL, Zerat L, et al. The role of Pipelle Mark II sampling in endometrial disease diagnosis. Eur J Obstet Gynecol Reprod Biol 2007;134:233-7.

38. Madari S, Al-Shabibi N, Papalampros P, et al. A randomised trial comparing the $\mathrm{H}$ Pipelle with the standard Pipelle for endometrial sampling at 'no-touch' (vaginoscopic) hysteroscopy. BJOG 2009;116:32-7.

39. Ngu SF, Cheung VY, Pun TC. Randomized study of vaginoscopy and $\mathrm{H}$ Pipelle vs traditional hysteroscopy and standard Pipelle. J Minim Invasive Gynecol 2012;19:206-11.

40. Guido RS, Kanbour-Shakir A, Rulin MC, et al. Pipelle endometrial sampling. Sensitivity in the detection of endometrial cancer. J Reprod Med 1995;40:553-5. 
41. Clark TJ, Mann CH, Shah N, et al. Accuracy of outpatient endometrial biopsy in the diagnosis of endometrial cancer: a systematic quantitative review. BJOG 2002;109:313-21.

42. Gungorduk K, Asicioglu O, Ertas IE, et al. Comparison of the histopathological diagnoses of preoperative dilatation and curettage and Pipelle biopsy. Eur J Gynaecol Oncol 2014;35:539-43.

43. Sanam M, Majid MM. Comparison the Diagnostic Value of Dilatation and Curettage Versus Endometrial Biopsy by Pipelle - a Clinical Trial. Asian Pac J Cancer Prev 2015;16:4971-5.

44. Seto MT, Ip PP, Ngu SF, et al. Positive predictive value of endometrial polyps in Pipelle aspiration sampling: a histopathological study of 195 cases. Eur J Obstet Gynecol Reprod Biol 2016;203:12-5.

45. Terzic M, Dotlic J, Vasiljevic M, et al. Endometrial polyps in infertility patients: The first study of their clinical characteristics. Clin Exp Obstet Gynecol 2018;45:692-8.

46. Ilavarasi CR, Jyothi GS, Alva NK. Study of the Efficacy of Pipelle Biopsy Technique to Diagnose Endometrial

Cite this article as: Terzic MM, Aimagambetova G, Terzic S, Norton M, Bapayeva G, Garzon S. Current role of Pipelle endometrial sampling in early diagnosis of endometrial cancer. Transl Cancer Res 2020;9(12):7716-7724. doi: 10.21037/ tcr.2020.04.20
Diseases in Abnormal Uterine Bleeding. J Midlife Health 2019;10:75-80.

47. Leitao MM Jr, Kehoe S, Barakat RR, et al. Accuracy of preoperative endometrial sampling diagnosis of FIGO grade 1 endometrial adenocarcinoma. Gynecol Oncol 2008;111:244-8.

48. Elsandabesee D, Greenwood P. The performance of Pipelle endometrial sampling in a dedicated postmenopausal bleeding clinic. J Obstet Gynaecol 2005;25:32-4.

49. Ben-Baruch G, Seidman DS, Schiff E, et al. Outpatient endometrial sampling with the Pipelle curette. Gynecol Obstet Invest 1994;37:260-2.

50. Aue-Aungkul A, Kleebkaow P, Kietpeerakool C. Incidence and risk factors for insufficient endometrial tissue from endometrial sampling. Int J Womens Health 2018;10:453-7.

51. Adambekov S, Goughnour SL, Mansuria S, et al. Patient and provider factors associated with endometrial Pipelle sampling failure. Gynecol Oncol 2017;144:324-8. 